\title{
AN INTEGRAL EQUATION WITH SYMMETRIC KERNELS*
}

BY T. S. PETERSON

It is the purpose of this note to investigate conditions necessary and sufficient for the solution of the integral equation

$$
\int_{a}^{b} A(x, s) X(s, y) d s+\int_{a}^{b} X(x, s) B(s, y) d s=C(x, y),
$$

where the kernels $A(x, y)$ and $B(x, y)$ are considered to be symmetric. We further restrict our functions of two variables to be continuous throughout the fundamental interval $(a, b)$.

An equation of the type (1) will not in general admit a solution. However, under certain quite restrictive conditions on the function $C(x, y)$, a solution may be obtained. To determine these conditions, we may readily verify from the classical theory of integral equations that every function $C(x, y)$, for which a function $X(x, y)$ exists such that $(1)$ is true, is developable in a uniformly convergent series

$$
C(x, y)=\sum_{i=1}^{\infty}\left\{\frac{\alpha_{i}(x) \bar{\alpha}_{i}(y)}{\alpha_{i}}+\frac{\bar{\beta}_{i}(x) \beta_{i}(y)}{\beta_{i}}\right\}
$$

where

(3) $\quad \bar{\alpha}_{i}(y)=\int_{a}^{b} \alpha_{i}(s) X(s, y) d s, \quad \bar{\beta}_{i}(x)=\int_{a}^{b} X(x, s) \beta_{i}(s) d s$,

and where $\left\{\alpha_{i}, \alpha_{i}(s)\right\}$ and $\left\{\beta_{i}, \beta_{i}(s)\right\}$ are the characteristic values and characteristic functions of the kernels $A(x, y)$ and $B(x, y)$, respectively. To justify this conclusion, it is sufficient to note that the series for the iterated kernel

$$
A^{(2)}(x, y)=\sum_{i=1}^{\infty} \frac{\alpha_{i}(x) \alpha_{i}(y)}{\alpha_{i}{ }^{2}}, \quad A^{(2)}(x, x)=\sum_{i=1}^{\infty} \frac{\alpha_{i}{ }^{2}(x)}{\alpha_{i}{ }^{2}},
$$

converge uniformly and absolutely, which, in view of the boundedness of $\sum_{i=1}^{\infty} \bar{\alpha}_{i}{ }^{2}(y)$, implies the uniform and absolute

* Presented to the Society, March 18, 1933. 
convergence of $\sum_{i=1}^{\infty} \alpha_{i}(x) \bar{\alpha}_{i}(y) / \alpha_{i}$. A similar argument applies for the other terms of (2).

This observation shows us that a necessary condition for a solution of the equation (1) under our hypotheses is that $C(x, y)$ be expressible linearly in terms of the characteristic functions of the kernels $A(x, y)$ and $B(x, y)$.

In the following theorems we shall have occasion to refer to a characteristic root of (1). If $\alpha_{i}$ and $\beta_{i}$ are the respective characteristic values of the symmetric kernels $A(x, y)$ and $B(x, y)$ and if $\alpha_{i}=-\beta_{k}($ any $i$ and any $k)$, we say that $\alpha_{i}\left(\right.$ or $\left.-\beta_{k}\right)$ is a characteristic root with respect to these kernels.

We may then state the following theorem.

Theorem 1. Assuming that (1) has no characteristic roots and that $C(x, y)$ has the necessary form

$$
C(x, y)=\sum_{i=1}^{\infty}\left\{\alpha_{i}(x) A_{i}(y)+B_{i}(x) \beta_{i}(y)\right\},
$$

then if the series

$$
\sum_{i=1}^{\infty}\left\{\alpha_{i} \alpha_{i}(x) \bar{A}_{i}(y)+\beta_{i} \bar{B}_{i}(x) \beta_{i}(y)\right\}
$$

is uniformly convergent, where $\bar{A}_{i}(y)$ and $\bar{B}_{i}(x)$ are defined by

$$
\left\{\begin{array}{l}
A_{i}(y)=\bar{A}_{i}(y)+\alpha_{i} \int_{a}^{b} \bar{A}_{i}(s) B(s, y) d s \\
B_{i}(x)=\bar{B}_{i}(x)+\beta_{i} \int_{a}^{b} A(x, s) \bar{B}_{i}(s) d s
\end{array}\right.
$$

it is a solution of (1).

This theorem is established directly by the substitution of (5) into (1) and noting by our hypothesis on the characteristic roots that the functions $\bar{A}_{i}(y)$ and $\bar{B}_{i}(x)$ are determined uniquely by (6).

It is evident that a more general solution of (1) may be obtained by adding to (5) any non-vanishing solutions of the equation

$$
\int_{a}^{b} A(x, s) Z(s, y) d s+\int_{a}^{b} Z(x, s) B(s, y) d s=0 .
$$


A treatment of the non-vanishing solutions of equations essentially of the form (7) has been made by Lauricella.* We wish here only to point out the following result.

THEOREM 2. If (7) has no characteristic roots, then all nonvanishing solutions of (7) have the property

$$
\begin{cases}\int_{a}^{b} \alpha_{i}(s) Z(s, y) d s=0, & (i=1,2, \cdots, n), \\ \int_{a}^{b} Z(x, s) \beta_{i}(s) d s=0, & (i=1,2, \cdots, n) .\end{cases}
$$

We may verify this theorem at once on multiplying (7) by $\alpha_{i}(x)$, integrating with respect to $x$, and applying

$$
\alpha_{i}(x)=\alpha_{i} \int_{a}^{b} A(x, s) \alpha_{i}(s) d s
$$

This gives us

$$
\left\{\int_{a}^{b} \alpha_{i}(s) Z(s, y) d s\right\}+\alpha_{i} \int_{a}^{b}\left\{\int_{a}^{b} \alpha_{i}(s) Z(s, t) d s\right\} B(t, y) d t=0 ;
$$

and hence

$$
\int_{a}^{b} \alpha_{i}(s) Z(s, y) d s=0 .
$$

A similar procedure establishes the second equation of (8).

Let us now consider the case in which there exist characteristic roots of (1). On multiplying (1) by $\alpha_{i}(x) \beta_{k}(y)$, (where $\alpha_{i}=-\beta_{k}$ ), and integrating on $x$ and $y$, we obtain further necessary conditions on the coefficients of $C(x, y)$, namely,

$$
\int_{a}^{b} A_{i}(s) \beta_{k}(s) d s+\int_{a}^{b} B_{k}(s) \alpha_{i}(s) d s=0 .
$$

With respect to these conditions, let us assume for the moment that the coefficients $B_{k}(s)$ are assigned arbitrarily and that the

* See Lauricella, Sopra le funzioni permutabili di $2^{a}$ specie, Lincei Rendconti, 1* Sem., (1913). 
$A_{i}(s)$ are determined by (10). This means that the $A_{i}(s)$ will have the form

$$
\begin{aligned}
A_{i}(s) & =\sum_{k} a_{i k} \beta_{k}(s)+A_{i}^{\prime}(s), \\
a_{i k} & =-\int_{a}^{b} \alpha_{i}(t) B_{k}(t) d t,
\end{aligned}
$$

where the summation is taken over all $k$ for which $\alpha_{i}=-\beta_{k}$, and where $A_{i}^{\prime}(s)$ is an arbitrary function which is orthogonal to all the corresponding $\beta_{k}(s)$. With this change we may rewrite the form of $C(x, y)$ as

$$
\begin{aligned}
C(x, y) & =\sum_{i=1}^{\infty}\left\{\alpha_{i}(x)\left[\sum_{k} a_{i k} \beta_{k}(y)+A_{i}^{\prime}(y)\right]+B_{i}(x) \beta_{i}(y)\right\} \\
& =\sum_{i=1}^{\infty}\left\{\alpha_{i}(x) A_{i}^{\prime}(y)+B_{i}^{\prime}(x) \beta_{i}(y)\right\} .
\end{aligned}
$$

Now considering the conditions (10) for the coefficients of this new form of $C(x, y)$, we have at once, since $\int_{a}^{b} A_{i}^{\prime}(s) \beta_{k}(s) d s=0$, the additional relation

$$
\int_{a}^{b} B_{k}^{\prime}(s) \alpha_{i}(s) d s=0 .
$$

For this reason we may assume, without loss of generality, that a necessary condition for the existence of a solution to equation (1) is that the function $C(x, y)$ have the form (4), where

$$
\int_{a}^{b} A_{i}(s) \beta_{k}(s) d s=0, \quad \int_{a}^{b} \alpha_{i}(s) B_{k}(s) d s=0
$$

for all $i$ and $k$ such that $\alpha_{i}=-\beta_{k}$. We may then generalize Theorem 1 to read as follows.

THEOREM $1^{\prime}$. Let the function $C(x, y)$ have the necessary form (4) satisfying the additional conditions (13); then if the series (5) is uniformly convergent, it is a solution of (1).

The only point in question with respect to the refinements made in the above theorem is whether the equations (6) will always permit a solution. We observe, however, that for $\alpha_{i}=-\beta_{k}$ the necessary and sufficient conditions for the solution of (6) are precisely the conditions (13). 
Considering the equation (7) with the introduction of characteristic roots, we have a somewhat different situation. Consider, for example, that $\alpha_{i}=-\beta_{k}$. Multiplying (7) by $\alpha_{i}$, we have

$$
\alpha_{i} \int_{a}^{b} A(x, s) Z(s, y) d s=\beta_{k} \int_{a}^{b} Z(x, s) B(s, y) d s .
$$

Multiplying (14) by $\alpha_{i}(x)$ and $\beta_{k}(y)$ separately and integrating, we have by (9)

$$
\left\{\begin{array}{l}
\int_{a}^{b} \alpha_{i}(s) Z(s, y) d s=\beta_{k} \int_{a}^{b} \int_{a}^{b} \alpha_{i}(s) Z(s, t) B(t, y) d s d t \\
\int_{a}^{b} Z(x, t) \beta_{k}(t) d t=\alpha_{i} \int_{a}^{b} \int_{a}^{b} A(x, s) Z(s, t) \beta_{k}(t) d s d t
\end{array}\right.
$$

These equations imply that

$$
\begin{aligned}
& \int_{a}^{b} \alpha_{i}(s) Z(s, y) d s=\sum_{k} c_{k} \beta_{k}(y), \\
& \int_{a}^{b} Z(x, t) \beta_{k}(t) d t=\sum_{i} c_{i}^{\prime} \alpha_{i}(x),
\end{aligned}
$$

where the summations extend over all $k$ and $i$ respectively for which $\alpha_{i}=-\beta_{k}$. These conditions on $Z(x, y)$ lead us to the following generalization of Theorem 2 .

THEOREM 2'. All non-vanishing solutions of (7) are of the form

$$
\sum_{i, k} c_{i k} \alpha_{i}(x) \beta_{k}(y)+Z(x, y),
$$$$
\left(c_{i k}=\text { const. }\right) \text {, }
$$

where $i$ and $k$ range over all indices having the property $\alpha_{i}=-\beta_{k}$, and where $Z(x, y)$ satisfies ( 7$)$.

Clearly the solution, (5), of (1) is made more general by the addition of terms (16) and the sufficiency as a solution is obvious.

Institute for Advanced Study 